\title{
EchoGéo
}

ECHOGEO $31 \mid 2015$

Glocal Ethiopia

\section{Le musée Konso au cœur de l'arène : quand les courtiers en développement (re)dessinent les contours du champ politique éthiopien}

\section{Chloé Josse-Durand}

\section{(2) OpenEdition}

Journals

Édition électronique

URL : https://journals.openedition.org/echogeo/14144

DOI : $10.4000 /$ echogeo.14144

ISSN : 1963-1197

Éditeur

Pôle de recherche pour l'organisation et la diffusion de l'information géographique (CNRS UMR 8586)

Référence électronique

Chloé Josse-Durand, « Le musée Konso au cœur de l'arène : quand les courtiers en développement (re)dessinent les contours du champ politique éthiopien », EchoGéo [En ligne], 31 | 2015, mis en ligne le 10 avril 2015, consulté le 31 juillet 2021. URL : http://journals.openedition.org/echogeo/14144 ; DOI https://doi.org/10.4000/echogeo.14144

Ce document a été généré automatiquement le 31 juillet 2021.

EchoGéo est mis à disposition selon les termes de la licence Creative Commons Attribution - Pas d'Utilisation Commerciale - Pas de Modification 4.0 International (CC BY-NC-ND) 


\title{
Le musée Konso au cœur de l'arène : quand les courtiers en développement (re)dessinent les contours du champ politique éthiopien
}

\author{
Chloé Josse-Durand
}

Je tiens à remercier le Centre Français des Études Éthiopiennes d'Addis-Abeba pour son soutien tant logistique que financier qui m'aura permis de réaliser plusieurs missions de recherche en Éthiopie entre 2008 et 2012, ainsi que Jean-Nicolas Bach pour les précieuses discussions autour du lien ténu qui existe entre la création du musée Konso et l'activation des catégories officielles de l'Éthiopie fédérale.

\section{Introduction}

1 Depuis le milieu des années 2000, les politiques publiques du gouvernement éthiopien se concentrent sur la préservation du patrimoine culturel, matériel et immatériel, ce qui s'est traduit par une multiplication des musées dans le pays ${ }^{1}$. Cette tendance a tout particulièrement touché la Southern Nations, Nationalities and Peoples' Region (SNNPR), l'État souvent considéré comme le « laboratoire ethnique » de l'Éthiopie fédérale, où un musée ethnographique a été ouvert au public dans la petite ville de Konso-karat en 2009, devançant l'inauguration prochaine d'un musée national du café à Bonga, dans la région Kaffa.

2 Ces projets muséaux ne peuvent être analysés uniquement comme le signe d'un intérêt ou une sensibilisation croissante pour la conservation: le patrimoine fonctionne comme un outil au service d'un projet politique de reconstruction de l'identité éthiopienne du parti au pouvoir, l'Ethiopian Party Revolutionnary Democratic Front (EPRDF). Les acteurs tant locaux, nationaux et internationaux qui prennent part aux 
processus de conservation, d'expertise et à la mise en place de projets contribuent à répondre aux objectifs bien souvent politiques à l'origine des politiques publiques du patrimoine. Les acteurs internationaux participent activement à ce phénomène: sollicités à la fois en tant que bailleurs de fonds et experts, ils jouent un rôle décisif dans la mise en œuvre des politiques publiques du gouvernement et s'inscrivent dans une nouvelle échelle, celle du glocal. Comment comprendre les jeux de pouvoir cristallisés par ces projets de développement alors qu'ils semblent échapper à l'État en créant un lien direct entre le local et l'international ? Ce type de rapport implique-t-il une moindre emprise de l'État éthiopien ou au contraire, renforce-t-il son caractère autoritaire? Dans quelle mesure cette nouvelle donne permet-elle à des acteurs locaux-guides touristiques, personnels du wereda ${ }^{2}$, chefs de projets, autorités traditionnelles-de développer des stratégies spécifiques de contournement de l'autoritarisme d'État?

3 En partant de l'hypothèse que les projets culturels mis en place au niveau local correspondent à une décompression autoritaire plus qu'à une libéralisation effective ${ }^{3}$, cet article se propose d'interroger les volontés d'emprise ou de ré-emprise de l'État éthiopien autour de la question de la mise en valeur d'un patrimoine national. Dans un premier temps, on cherchera à mettre en évidence les modalités selon lesquelles la problématique patrimoniale nous amène à penser les acteurs des institutions muséales comme parties prenantes d'enjeux plus larges. Le nouveau paradigme qui a accompagné la campagne du Millénium, renouveler les bases du vivre-ensemble en redéfinissant la rhétorique de l' "éthiopianité » héritée de l'ère impériale (Bureau, 1987; Gascon, 1995), a donné naissance à des politiques publiques du patrimoine ancrées dans un vaste programme de reconstruction nationale (Bach, 2013b). Dans cette perspective, le musée Konso, présenté par la conservatrice du Musée National d'Addis-Abeba comme le premier «single-ethnic Museum » d'Éthiopie, nous permettra de mieux comprendre les priorités du Developmental State éthiopien. Issu d'une recherche entre des partenaires scientifiques français et éthiopiens mais également d'une entreprise exogène de la coopération française soutenue par le musée du Quai Branly et l'UNESCO, il fonctionne aujourd'hui comme la vitrine d'un fédéralisme ethnique efficace, symbole de la démocratisation progressive du pays entreprise sous la houlette de l'EPRDF. Dans le même temps, l'usage stratégique des normes internationales par le gouvernement et la course au classement UNESCO consolident les canaux de contrôle du parti au pouvoir tout en diversifiant les modalités de diffusion de l'image positive d'un État développementaliste démocratique. Néanmoins, des acteurs particuliers émergent de ce type de projet de développement. Dotés de stratégies propres et d'un potentiel d'action, les «courtiers en développement » restructurent les rapports de pouvoir entre le local et le national en traitant directement avec des acteurs internationaux (Bierschenk \& Sardan, 2000). Ils jouent pourtant un rôle ambivalent : en participant à la valorisation du patrimoine culturel éthiopien, ils sont également les acteurs de la légitimation tant nationale qu'internationale de l'idéologie développementaliste et des directives mises en place par le gouvernement. 


\section{De l' « Éthiopie sans monuments » à la « Shining Ethiopia » (A. Gascon)}

\section{Lâge d'or du patrimoine culturel}

4 Tantôt qualifiée par le gouvernement de musée à ciel ouvert ou désignée comme le «berceau de l'humanité » en raison de nombreuses découvertes archéologiques (parmi lesquelles le squelette de Lucy dans la basse vallée de l'Awash), l'Éthiopie est souvent présentée comme un musée des peuples par les autorités éthiopiennes. Cette image fait pourtant écho à un héritage controversé : Carlo Conti Rossini, ancien administrateur colonial italien, est le premier à avoir fait le portrait de l'Abyssinie comme un « museo dei popoli ». Les médias et notamment la presse, le journal gouvernemental The Ethiopian Herald en tête, égrènent aujourd'hui cette idée, qui est devenue un véritable slogan touristique se déclinant selon les États régions. Le Bureau du tourisme du Tigré fait campagne sur le thème "Tigray, the open-air museum ", mélangeant sur quelques cartes postales scènes de la vie quotidienne, coiffures traditionnelles, églises et peintures chrétiennes (illustrations $1 \mathrm{a}$ et $1 \mathrm{~b}$ ). De la même façon, on retrouve l'idée d'une "Éthiopie, mosaïque des peuples » sur les brochures du Bureau régional du Tourisme de la SNNPR. Elle est également reprise lors de discours politiques et donne naissance à une célébration, le Nations, Nationalities and People's Day, au cours de laquelle le premier Ministre Méles Zénawi confie aux peuples d'Éthiopie la lourde et symbolique tâche de représenter la Renaissance ${ }^{4}$ éthiopienne (Bach, 2013b). L'unité du pays se fera dans la diversité des Nations, Nationalities and Peoples selon le mot d'ordre de l'EPRD : "Our diversity is our beauty, and our beauty is our unity ».

\section{Illustrations $1 \mathrm{a}$ et $1 \mathrm{~b}$ - Cartes postales du Bureau de la Culture du Tigray présentant l'Éthiopie} comme un « musée à ciel ouvert »

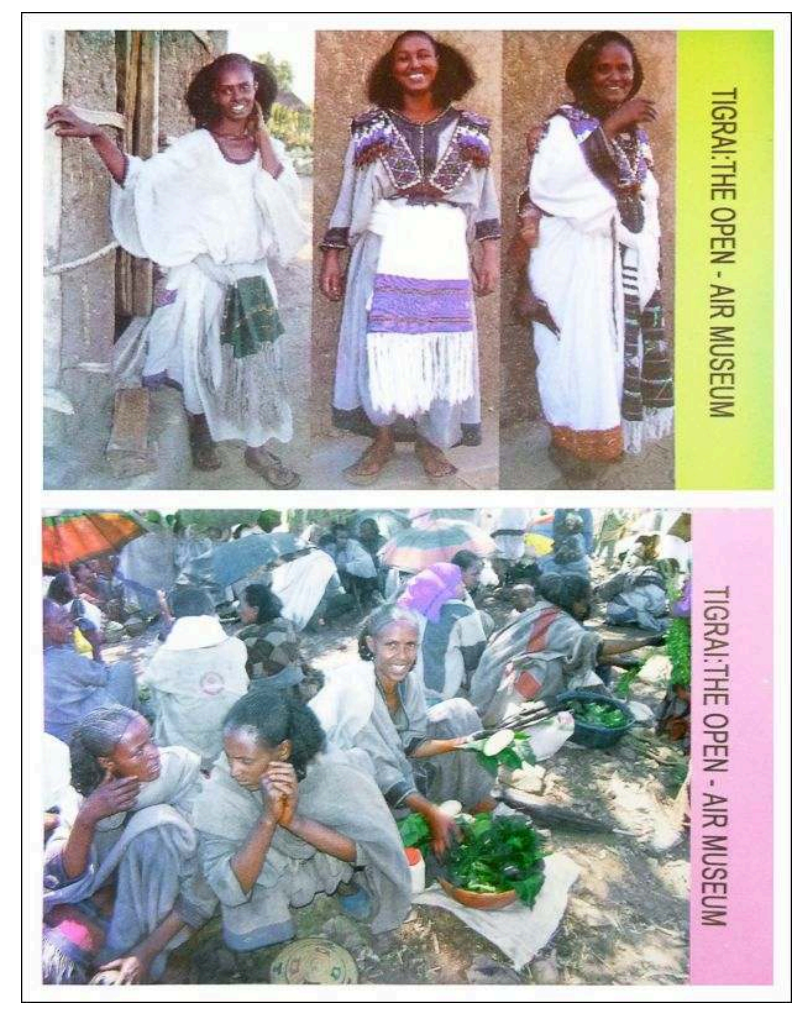




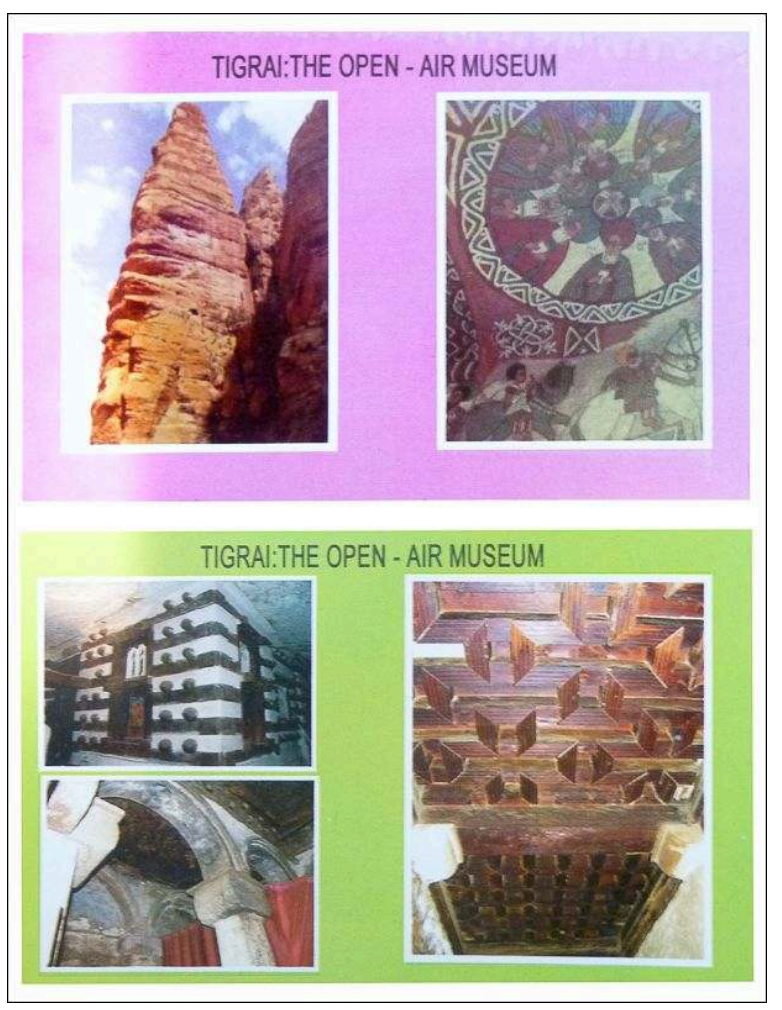

Montages réalisés par C. Josse-Durand.

5 La mise en place de ce nouveau paradigme s'explique, selon Jean-Nicolas Bach, par la double crise que traverse l'État éthiopien : une crise « de » l'État, qui se traduit par une remise en cause de l'État éthiopien, et une crise « dans » l'État, c'est à dire une remise en cause du régime et du gouvernement éthiopien mais pas de l'État en lui-même (Bach, 2011). Les critiques et le scepticisme qui ont accompagné la mise en place du régime ethnofédéral en 1994 participent activement à cette double remise en question de l'État (Assefa Fiseha, 2006; Abbink \& Hagmann, 2013). Les manifestations inédites qui font suite aux élections de 2005, au cours desquelles la population se prononce en faveur de l'opposition et remettent en cause les fondements du fédéralisme ethnique, obligent le gouvernement à explorer de nouvelles façons de gérer les communautés infra-nationales: des politiques culturelles spécifiques voient le jour, ayant pour délicate mission de gommer l'image communément admise d'un État éthiopien administré par des cadres tigréens et dirigé par une élite Amhara (Abbink, 2006). Cette politique culturelle s'accompagne d'un ensemble de réformes et d'événements symbolisant un changement d'ère, alors que le parti au pouvoir depuis plus de vingt ans, ses cadres dirigeants et son premier ministre Méles Zenawi, contrôlent les institutions du pays, musellent la société civile et intimident l'opposition.

6 Le Millénium, la campagne de célébration du passage à l'an $2000^{5}$, a été l'occasion pour l'EPRDF de rendre visible ce discours de la Renaissance par une succession d'événements, de commémorations, de présentations de projets de long terme et de promesses politiques qui ont rythmé la vie de la Nation de septembre 2007 à septembre 2008 (Bach, 2009). Depuis le Millénium, le slogan "unis dans la diversité » tend à s'affirmer jusqu'à devenir une sorte de mot d'ordre des politiques publiques du patrimoine en Éthiopie. Soudainement reconnue comme une région riche d'une culture encore peu exploitée, le Sud de l'Éthiopie, jusqu'alors considéré comme le grenier à 
grain de l'époque impériale, habité par des tribus sans fondement chrétien qui parlaient un amharique sommaire, ne mangeaient pas d'injera et n'attiraient que les anthropologues et les touristes en quête d'aventure suscite un intérêt sans précédent (Gascon, 1995; Planel, 2008). En faisant la publicité de cette "Éthiopie sans monuments " évoquée en 1995 par le géographe Alain Gascon, la valorisation du patrimoine culturel s'est révélée être un outil efficace afin de dépasser l'opposition traditionnelle qui existe entre l'Éthiopie du Nord, celle des hauts plateaux chrétiens et des sites UNESCO mondialement connus comme Lalibela, Gondar, Yeha ou Aksum, et les communautés marginalisées du Sud (Gascon, 1995; Bridonneau, 2013). L'effacement progressif, en surface, de ce clivage historique sur lequel s'est construite la " Grande Éthiopie » semble être, aux yeux de l'EPRDF, l'une des conditions de l'émergence d'une «Shining Ethiopia» qui ne serait plus rurale mais urbaine (Gascon, 2008), d'un Middle Income Country au sens de la Banque Mondiale qui s'appuierait sur ses «classes moyennes » afin d'assurer croissance et stabilité à l'horizon 2025 (Lefort, 2013 ; Nallet, $2014)^{6}$.

\section{"Ethiopia leading in Africa » : construire l'image d'un État développeur}

7 Si le patrimoine est pensé comme un nouvel outil politique par le parti au pouvoir, il est également pensé comme un levier économique. Afin de pallier le manque d'investissements directs à l'étranger, le gouvernement cherche à développer son offre touristique pour attirer de plus en plus de visiteurs internationaux mais également domestiques (Mengistu Gobezie, 2008). Un certain nombre d'initiatives en témoignent : des manifestations culturelles, mais également l'affichage public ainsi que le message véhiculé par ce dernier. En affichant sa volonté d'associer le peuple éthiopien aux grands projets d'État, le parti au pouvoir renforce par la même occasion la portée de l'Abyotawi democracy ou la "démocratie révolutionnaire", une idéologie marxiste à l'éthiopienne qui s'oppose au néo-libéralisme et à l'impérialisme (Vestal, 1999; Abbink \& Hagmann, 2013 ; Bach, 2013a) illustrée par les affiches jalonnant les rues d'AddisAbeba présentant Meles Zenawi guidant les peuples d'Éthiopie vers le développement et un avenir radieux. C'est également pour cette raison que le Millénium, en tant que vaste mise en scène de la Renaissance tant identitaire qu'économique du pays, s'est accompagné d'investissements importants $d u$ gouvernement dans des projets culturels dont participe la création, en décembre 2009, d'un premier musée ethnographique dans la SNNPR. Ces politiques du patrimoine contribuent à donner un nouveau visage à la nation éthiopienne forte de sa diversité et unie autour de certaines valeurs, dans une tentative de refaçonner le visage du parti au pouvoir et de la classe politique ${ }^{7}$.

8 Le gouvernement utilise également le patrimoine culturel éthiopien afin de répondre à une autre de ses priorités: promouvoir une nouvelle image de l'Éthiopie à l'international et la propulser au-devant de la scène comme l'une des cinq premières destinations touristiques d'Afrique (illustration 2). 
Illustration 2- Première et dernière de couverture d'une brochure officielle du gouvernement associant le classement au patrimoine mondial de Konso à l'idée d'une "Ethiopia leading in Africa »

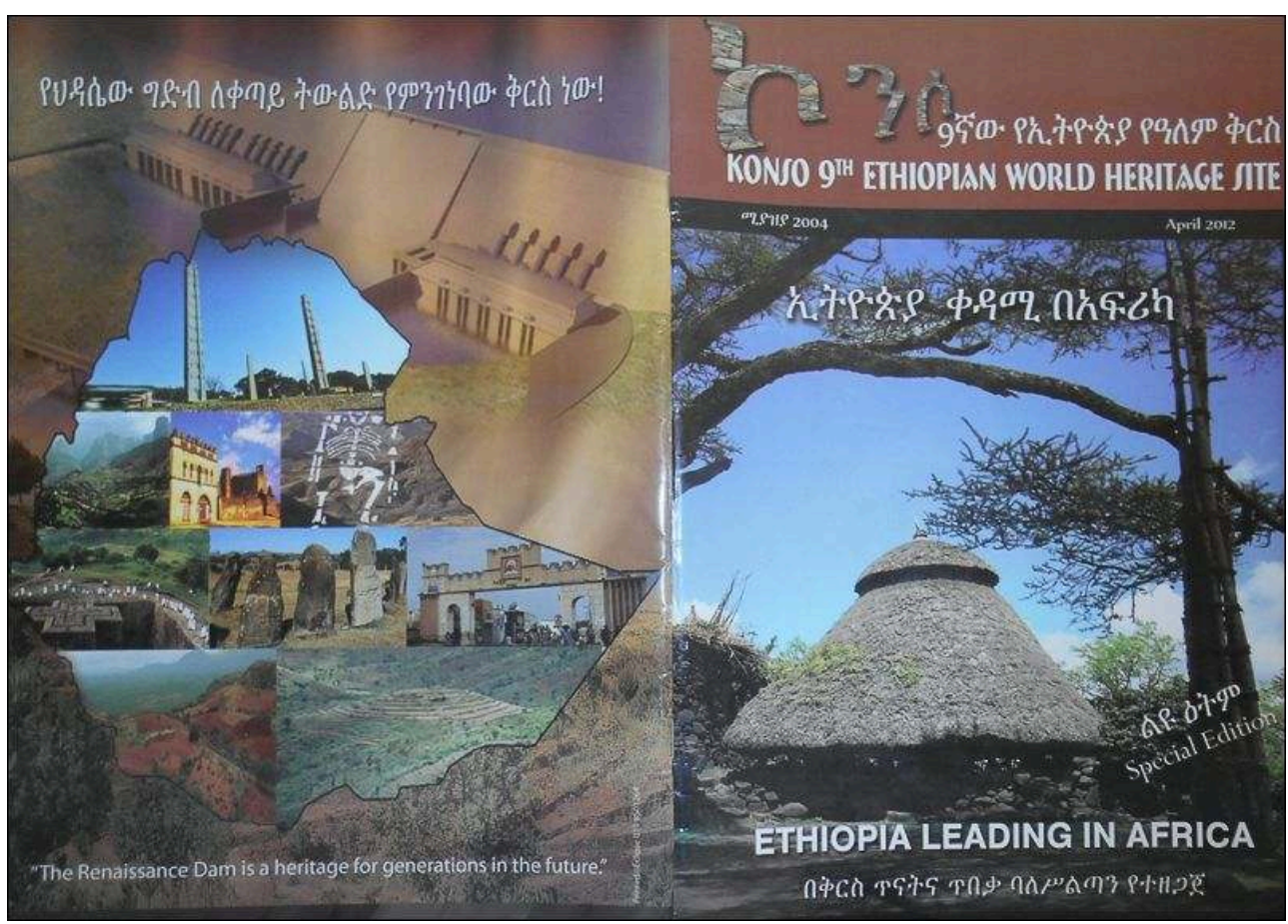

Auteur: C. Josse-Durand.

9 Le développement du tourisme culturel dans l'ensemble des régions, où l'on retrouve des campagnes publicitaires similaires, se couple d'un usage stratégique des normes internationales. Les définitions de l'UNESCO sont reprises comme cadres de référence par les responsables du Musée National d'Addis-Abeba mais aussi par les acteurs locaux de ces politiques ${ }^{8}$. Dans cette perspective, le classement de Konso en tant que paysage culturel en juin 2011 est un exemple parmi d'autres de la "course au classement UNESCO » que mène l'Éthiopie. Entre 1977, l'année où elle signe la Convention concernant la protection du patrimoine mondial culturel et naturel avec l'UNESCO, et 2006, elle a en effet classé 9 sites au patrimoine mondial, ce qui la propulse au premier rang des États-partie d'Afrique (Chaudron, 2008). Cette logique de classement et l'ouverture du pays à la manne financière du tourisme culturel révèlent une nette stratégie d'intégration de l'Éthiopie au circuit international.

10 Il s'agit donc de redorer l'image du pays à l'international tout en répondant aux objectifs internes du Growth and Transformation Plan pour 2015, un plan quinquennal ambitieux visant à augmenter fortement la croissance économique annuelle de l'Éthiopie en favorisant son industrialisation, l'essor du secteur des services et la mise en place de grands projets d'infrastructures représentés par le barrage du Nil ou Grand Ethiopian Renaissance Dam (Nallet, 2014 ; illustration 2). Ainsi, le développementalisme devient une idéologie plus qu'une politique économique, visant à légitimer le contrôle de l'État et ses actions de répression contre l'opposition et la société civile, présentant les auteurs de tout acte de réprobation comme les ennemis du développement (Aalen, 2006). Or, l'État éthiopien n'a pas les moyens de mener tous ces objectifs de front. Il s'appuie donc sur des acteurs de la coopération internationale comme l'UNESCO « pour briller à l'international » mais également sur des partenariats bilatéraux diplomatiques 
(Ambassade de France), scientifiques (Centre Français des Études Éthiopiennes - CFEE) ou institutionnels (Musée du Quai Branly).

\title{
Quand le local reconfigure le national : le musée Konso, « vitrine » du fédéralisme ethnique
}

\author{
Un projet culturel qui se fait le miroir d'une démocratisation bien \\ réelle
}

11 L'origine du musée Konso n'est pas directement liée aux priorités gouvernementales du Millénium : c'est avant tout le fruit d'une collaboration entre différentes institutions de la coopération internationale (UNESCO, Musée du Quai Branly, Ambassade de France, $C F E E$ ) qui sera récupérée par la suite à des fins politiques. Le projet naît dans les années 1990, d'un intérêt scientifique partagé par des chercheurs français et éthiopiens de l'Authority for Research and Conservation of the Cultural Heritage (ARCCH), pour la préservation des statues funéraires Konso, les Wakka, d'une détérioration rapide et d'un commerce illégal. Ces derniers déposent un premier projet de classement au patrimoine mondial de l'humanité auprès de l'UNESCO. En 2005, Stéphane Gompertz, alors Ambassadeur de France en Éthiopie, se passionne pour le patrimoine Konso lors d'une visite dans la région. Des fonds sont débloqués par le Service de Coopération et d'Action Culturelle, qui se tourne vers le CFEE et le Musée du Quai Branly à Paris pour une mission d'expertise (Bosc-Tiessé, 2009). Alors qu'il existe une volonté évidente de valoriser la culture Konso de la part du Bureau de la Culture du Konso Special wereda et de quelques personnalités locales, ce n'est que plus tardivement que les autorités éthiopiennes régionales s'intéressent à l'établissement d'un musée ethnographique au sein de la SNNPR (Roig, 2010). Le musée émerge finalement d'une coopération complexe et segmentaire, avec pour objectif de donner naissance à une "community based organization", c'est-à-dire à une structure ancrée au niveau local et à terme indépendante financièrement dont la mission serait de préserver et de présenter les différents aspects de la culture Konso et son patrimoine en danger.

12 En réalité, le musée ne remplit pas complètement ses objectifs initiaux : il joue un rôle clé dans une stratégie politique plus large ayant pour objet de mettre en scène un fédéralisme efficace. La muséographie, finalisée par Raphaël Roig alors stagiaire en coopération culturelle au CFEE, se divise en 2 espaces principaux : la galerie des wakka qui présente les pratiques funéraires, le système clanique et les classes d'âge Konso, et le grand tukul ${ }^{9}$ qui sert principalement de point d'accueil pour les visiteurs. L'équipe locale a ensuite réarrangé les panels explicatifs en fonction des objets (calebasses, outils agricoles, instruments de musique, bijoux, armes, jeux, etc.) qu'ils ont ajouté au parcours muséal sans qu'ils ne fassent l'objet d'une explication précise. Une coopérative artisanale a également été installée par la suite dans l'enceinte du musée, soulignant la vocation touristique du lieu. En réalité, le musée ne joue pas son double rôle d'institution locale et touristique. Cet échec se justifie sur la base de plusieurs constats: si la composition des panels exposés dans la galerie principale pose clairement la question du public auquel s'adresse le musée (une ligne seulement est écrite en konsigna tandis que l'anglais, le français puis l'amharique occupent graduellement les panels), les Éthiopiens ne représentent qu'un tiers des visiteurs accueillis par le musée en 2012, sans qu'aucun tarif préférentiel n'ait été mis en place 
pour les habitants de la région Konso ${ }^{10}$. La construction du Kanta lodge en face du musée, un hôtel de luxe copiant l'architecture des villages fortifiés voisins, n'est que l'un des éléments d'un projet touristique plus large qui génère de nombreux conflits : il n'est pas rare que les habitants des villages identifiés comme des étapes clés des visites touristiques au départ de Konso-Karat barricadent les entrées, malgré les négociations entamées par les guides locaux ${ }^{11}$. Enfin, le Konso Cultural Center financé par l'organisation non-gouvernementale (ONG) italienne Cooperazione Internationale Sud Sud (CISS) et l'Union Européenne attire de plus en plus de résidents depuis son ouverture en 2010, et notamment les jeunes générations qui ont à leur disposition une bibliothèque ainsi qu'un espace audio-visuel.

13 Rapidement, le musée a échappé à la maitrise de ses initiateurs extérieurs que sont l'Ambassade de France, le CFEE et le Musée du Quai Branly : force est de constater que le projet en lui-même participe de la "mise en scène " ${ }^{12}$ de la République fédérale et démocratique d'Éthiopie. S'il revient à la charge du wereda d'un point de vue financier, sa muséographie est mise sous tutelle du Musée National d'Addis-Abeba, qui apporte un soutien logistique en transférant une dizaine de bureaux depuis la capitale jusqu'au musée, sans pourtant y amener des objets susceptibles de compléter la collection existante. Avant même son inauguration, le journal éthiopien Capital présentait déjà le projet comme une étape clé du processus fédéral : «This project is hoped to provide an answer to some of the questions concerning cultural and identity needs of the contemporary Konso people, who are one of the many peoples in Southern Ethiopia participating in the building of national identity within a federalist framework ». Cette mainmise des acteurs d'État que sont le Musée National d'Addis Abeba, le Special wereda de Konso et le Bureau de la Culture régional d'Awasa s'explique par deux phénomènes: l'autoritarisme d'État et le contrôle que le gouvernement central maintient sur ses périphéries par le biais des différents échelons composant le système fédéral, ce qui rejoint la thèse affirmant que l'ethno-fédéralisme constitue un système politique de contrôle efficace (Planel \& De Poix, 2009; Assefa Fisseha, 2006 ; Abbink \& Hagmann, 2013). Ce projet de musée a donc permis à l'État éthiopien de matérialiser ses politiques publiques internes en périphérie avec l'aide d'acteurs de la coopération internationale (illustration 3). 


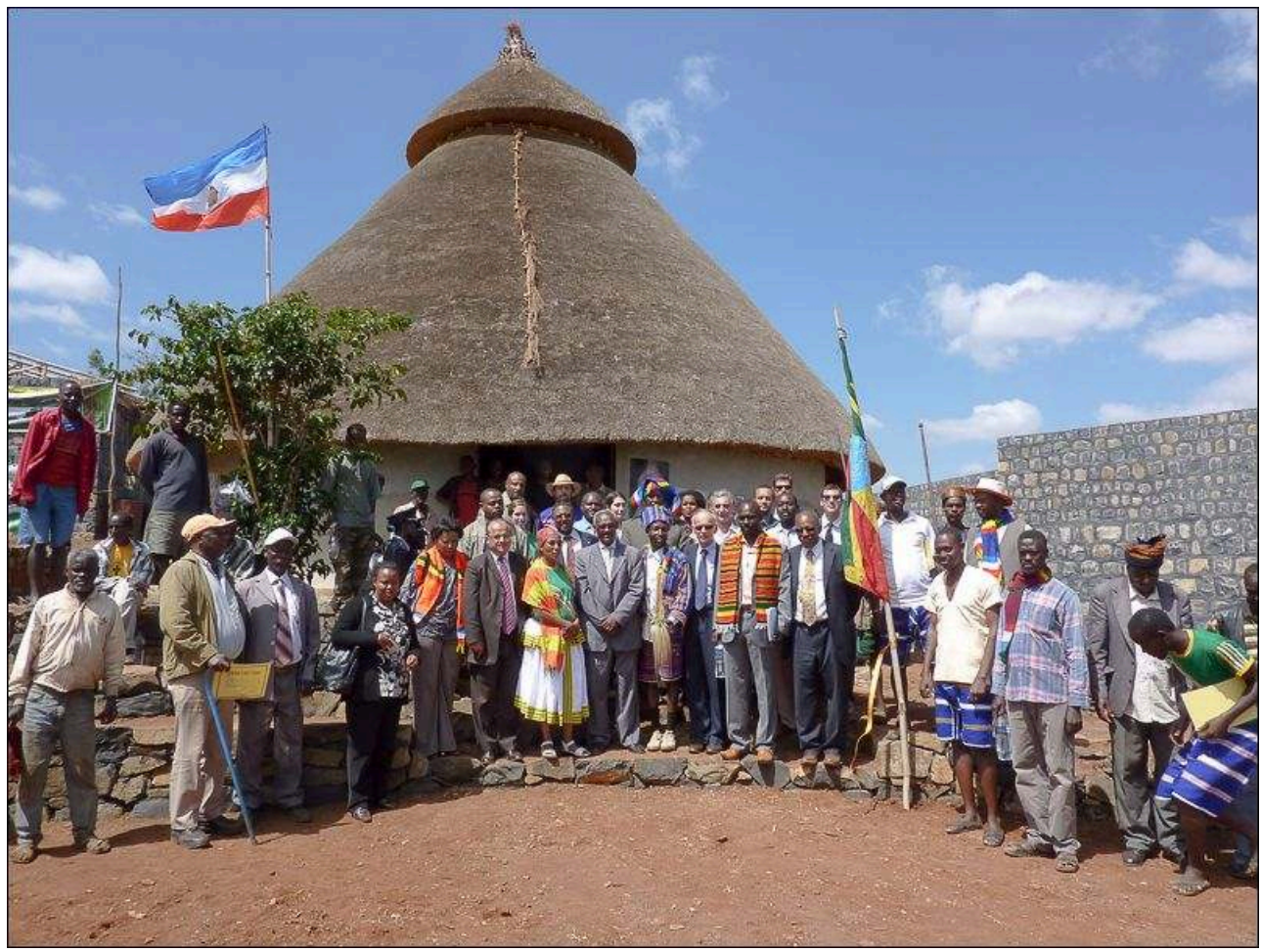

Le Secrétaire d'État pour la Culture et le Tourisme éthiopien, le directeur et l'équipe du CFEE, les experts du musée du Quai Branly, Stéphane Gompertz alors Ambassadeur de France en Éthiopie, le Directeur du Bureau Afrique du Ministère des Affaires Étrangères français, les représentants du Bureau de la Culture de la SNNPR et de Konso, les représentants des autorités patrimoniales éthiopiennes (I'ARCCH et le musée d'Addis-Abeba) et le Kalla Gesahegn, au centre de la photo - réunis devant le grand tukul du musée lors de son inauguration le 18 décembre 2009.

Auteur: M.-L. Derat.

Dans le même temps, cette coopération avec des autorités internationales de renom qui ont apporté un soutien financier, matériel et scientifique au projet (notamment l'UNESCO et le musée du Quai Branly) valide en quelque sorte l'hypothèse d'une démocratisation réelle $\mathrm{du}$ régime, alors que la censure persiste sur d'autres revendications, telles que l'association des autorités claniques au processus décisionnel, la redistribution des ressources aux communautés villageoises qui dressent de fait des barricades pour empêcher les visites, ou les aspirations de transparence et de bonne gouvernance (Asefa Fiseha, 2012; Planel, 2007; Asnake Kefale, 2009). En levant d'une certaine façon le contrôle strict d'État sur les projets de développement étrangers en général ${ }^{13}$, ce musée est devenu la bannière mobilisée par le gouvernement pour justifier de l'avancée des processus de décentralisation et de démocratisation.

\section{Catégoriser pour mieux contrôler : donner vie aux Nations, Nationalities and Peoples}

15 Au cœur des enjeux de gouvernance, le musée communautaire Konso fonctionne comme un projet politique national expérimenté à l'échelle locale et souligne la tension de longue date qui existe entre les objectifs nationaux et politiques de l'EPRDF (développement national, la stabilité, l'augmentation de la croissance économique du pays) et les demandes des minorités éthiopiennes en termes de droits constitutionnels, 
de reconnaissance culturelle et de développement local (Aalen, Pausewang \& Tronvoll, 2002).

16 En effet, la création du musée Konso s'inscrit dans des luttes de pouvoir latentes. Comme nous l'avons précisé auparavant, le gouvernement a imposé l'ethnie comme cadre pertinent de compréhension de la nation éthiopienne avec l'apparition d'une nouvelle rhétorique identitaire inclusive mise en avant par la campagne du Millénium. Pendant des siècles, les populations du Sud n'étaient pas partie prenante de la nation éthiopienne. Les Konso étaient de ceux-là : ils avaient alors le statut de gäbar ${ }^{14}$ et connurent de nombreuses disettes (Otto Shako, 2005 ; Merera Gudine, 2006). Annexée en 1890 par Ménélik II, la région n'a été intégrée que tardivement à l'Éthiopie impériale. À cette époque, il se dit que certains s'engagèrent volontairement dans l'armée italienne pour combattre l'Empereur (Vaughan, 2006). En ce sens, il faut questionner les rapports de pouvoir qui existent au sein de l'Éthiopie contemporaine en prenant en compte les tensions découlant des cadres ethno-fédéraux de la Constitution de 1994. Dans cette perspective, le musée Konso est pensé par le gouvernement comme un projet venant transcender les cadres d'appartenances primaires (la famille, le clan, l'ethnie) pour se constituer comme un espace d'affirmation de l'identité nationale. En établissant les catégories prédéterminées que sont les Nations, Nationalities and People, la Constitution fédérale a également été un moyen pour le gouvernement de catégoriser pour mieux contrôler. Néanmoins, ces catégories établies " par le haut », centrales dans la définition officielle de l'ethno-fédéralisme éthiopien, restent floues et participent de la perte de confiance dans les institutions, alimentant les critiques faites au système ethno-fédéral. En ce sens, le musée Konso fonctionne comme un véritable outil afin de donner vie aux catégories nationales et d'affirmer leur pertinence.

17 Ce musée assume une fonction symbolique primordiale en rappelant aux éthiopiens qu'être citoyen de la fédération implique d'être conscient des multiples appartenances de tout un chacun. L'exposition du patrimoine Konso légitime ainsi les cadres imposés par la Constitution et met en scène ce que les Nations, Nationalities and Peoples devraient être, bien plus qu'il ne reconnaît la singularité d'une communauté infra-nationale éthiopienne. Le musée n'est rien d'autre que la mise en scène plus large d'une possible réunification nationale par la culture. Amhara, Oromo, Somali, Konso, font partie d'une communauté politique unie: la fédération éthiopienne. Cela souligne le contrôle permanent de l'État éthiopien qui autorise sa population à s'exprimer ou à se définir uniquement selon les catégories imposées par la Constitution fédérale. En même temps que le gouvernement entretient l'illusion d'une démocratisation progressive, en pratique, une réforme territoriale organise la "recentralisation » des groupes définis en termes ethnolinguistiques. Dans le cours de l'année 2011, plusieurs Special wereda ${ }^{15}$ seront transformés en simples wereda pour se fondre dans des zones regroupant plusieurs groupes, remaniement récurrent visant au contrôle territorial de la SNNPR (Vaughan, 2006). Le Special wereda de Konso a disparu, il s'agit maintenant d'un wereda parmi d'autres, rattaché à la Segen Area People's Zone, nouveau centre administratif et politique de la région (illustration 4). 
Illustration 4 - : Sur la route de Konso-karat, un panneau signalant la nouvelle Segen Zone issue de la réforme territoriale des Special wereda

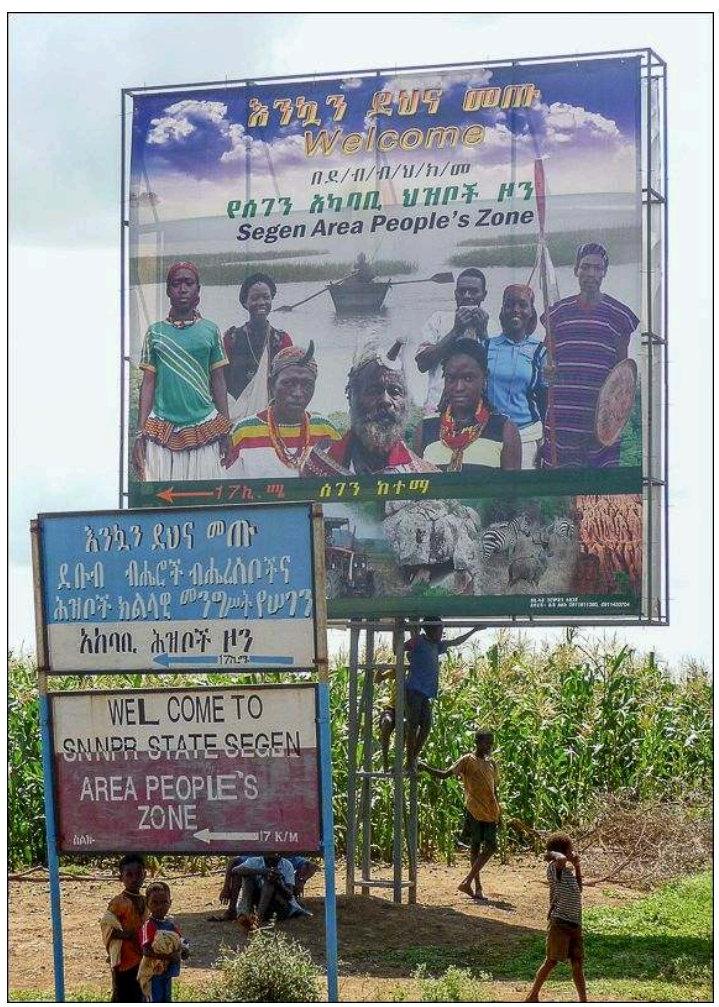

Auteur : C. Josse-Durand.

Cette réforme souligne bien que le musée Konso n'est pas la pierre de touche d'une décentralisation réelle ou encore un assouplissement des modalités de contrôle inhérentes au système ethno-fédéral en place, mais bien la vitrine d'une "démocratisation de façade » voulue par le parti au pouvoir (Planel, 2007). C'est en ce sens qu'on peut plutôt parler d'une décompression autoritaire que d'une réelle démocratisation du pays (Bayart, 1990 ; Médard, 1990 ; Camau \& Geisser, 2003).

\section{Le musée Konso ou la glocalité en pratique}

\section{Le Kalla Gesahegn et Dinoté Kusia : portraits de « courtiers en développement »}

Le glocal, cette relation ambivalente entre le local et l'international, modifie sensiblement l'articulation des réseaux autoritaires traditionnels en Éthiopie. Cette nouvelle dialectique du pouvoir se révèle dans les entretiens menés avec certaines personnalités de Konso qui s'imposent à travers la mise en place du musée. En effet, le projet de musée Konso, comme tout projet de développement, fonctionne comme une zone tampon entre l'État et ses périphéries, perçue comme un champ d'opportunité dont certains acteurs n'hésitent pas à s'emparer (Bierschenk \& Olivier de Sardan, 1998). Ces acteurs sont multiples et n'ont pas les mêmes trajectoires, profils ou objectifs : les histoires de personnes révèlent par exemple des enjeux de pouvoir entre les administrateurs du Special wereda de Konso et les représentants du pouvoir clanique $\left(\right.$ Pogall $\left.^{16}\right)$. On cherchera ici à questionner plus particulièrement le rôle d'une catégorie 
d'acteurs qui s'affirme avec la mise en place du musée: les «courtiers en développement $»$.

Ces « courtiers en développement ", au sens des anthropologues T. Bierschenk et J.-P. Olivier de Sardan, sont des acteurs sociaux implantés dans une " arène locale » au sein de laquelle ils obtiennent une importance politique en jouant de leurs ressources propres - être lettré, savoir parler anglais, avoir été guide touristique ou membre de l'administration locale. En contribuant à la préservation et la mise en valeur du patrimoine Konso, ils se constituent comme des intermédiaires nécessaires, drainant vers l'espace local des ressources relevant de l'aide au développement. Les stratégies mises en place par deux membres du comité scientifique informel du musée Konso, Dinoté Kusia et le Kalla Gesahegn ${ }^{17}$, sont particulièrement intéressantes en ce qu'elles révèlent les véritables enjeux de pouvoir qui accompagnent la création du musée. Dinoté Kusia se présente délibérément comme un personnage éminemment politique : en tant qu'administrateur du musée pendant les 6 premiers mois de son fonctionnement, il était en contact étroit avec les visiteurs et a utilisé son statut pour faire passer des messages politiques auprès des touristes mais aussi des médias internationaux. Le Kalla Gesahegn, chef de l'un des 9 clans structurant la société Konso, se présente au contraire comme le représentant spirituel de sa communauté. Il utilise le musée afin d'orienter la demande touristique vers son village et expliquer les conflits qui l'opposent au Special wereda de Konso, qui lui reproche de menacer l'autorité d'État en renforçant ses propres réseaux d'influence.

21 Dinoté Kusia, âgé d'une soixantaine d'années, est le premier administrateur du musée Konso et une personnalité bien connue localement pour avoir été instituteur, guide touristique et membre du wereda durant quelques années. Il intervient à tous les niveaux du projet, souhaitant s'affirmer comme un intermédiaire indispensable entre le Special wereda de Konso et les acteurs de la coopération française. Pour cela, il joue sur deux plans, se positionnant comme un acteur clé, mobilisant un discours appuyant sa légitimité à la fois "traditionnelle » en mettant avant ses liens de parenté avec le Kalla Gesahegn dont il affirme être le "grand oncle », et "moderne ", évoquant son ancienne fonction d'instituteur ainsi que son rôle pionnier au sein du Bureau de la culture de $\mathrm{Konso}^{18}$. C'est en partie cette fonction qui lui a permis d'accéder au statut de d'administrateur temporaire du musée Konso, avant qu'un directeur officiel ne soit nommé par le Special wereda. Pourtant, lors d'une de nos premières entrevues à Konso, il nous révèle que plusieurs membres du wereda ne soutiennent pas sa candidature à la direction du musée : " They want to push me from being director: they don't want a politic but a cultural director». Selon lui, le musée serait investi d'enjeux politiques cristallisant les rapports complexes qu'entretiennent les autorités traditionnelles et les autorités administratives du Special wereda, et plus largement l'opposition qui persiste en Éthiopie entre centre et périphéries (Asnake Kefale, 2009; Bach, 2011). Durant l'inauguration du musée en décembre 2009, il a été sollicité à de nombreuses reprises par les médias. Il aurait alors tenu un discours politique similaire, clamant que le gouvernement central écrase les volontés du peuple : «If the opposition would know that, they would change everything ». Bien que selon lui aucun officiel ou membre du wereda n'ait pu l'entendre à ce moment précis, il précise avec un soupçon de fierté: "They knew what I was saying». Au-delà du fait qu'il mentionne la culture comme un outil politique, il s'intéresse plus particulièrement à la rente générée par la mise en place d'un circuit touristique à Konso. Il fait régulièrement allusion à l'argent du tourisme, 
promis par le gouvernement aux communautés de chacun des villages Konso et qui n'a jamais été redistribué aux populations : "I'm not a politician but I'm mobilizing the people, I tell them the good things, that's all: ask the officials, the governement, the wereda your money!». Son discours dénonciateur est essentiellement centré sur les intentions politiques et économiques du gouvernement éthiopien qui accompagnent ce projet culturel. Bien que sa stratégie soit paradoxale - il se comporte comme un acteur politique sans se présenter comme tel - il dénonce la spoliation des populations locales, espérant ainsi s'attirer la sympathie et la confiance des observateurs étrangers malgré les suspicions quant à sa motivation première: récupérer une part de la rente patrimoniale auprès des touristes, des institutions et des chercheurs investis dans le projet $^{19}$.

22 Si l'on reprend le présupposé de Jeremy Boissevain, le courtier est partie prenante d'un système d'intermédiation où il joue habilement et activement avec un réseau de personnes reliées à un ensemble d'informations (Boissevain, 1979). Dinoté Kusia est donc un «entrepreneur» dont le capital serait constitué de relations personnelles assez développées pour lui permettre de s'instituer comme un " homme de réseau ». En échange de son aide aux experts, aux chercheurs sur le terrain ou encore aux autorités françaises, il attend et reçoit une "commission » qui prend le plus souvent la forme d'un don matériel : des vêtements pour sa femme et son jeune fils, un appareil photo numérique, de nouvelles lunettes, un téléphone portable, une mobylette pour gravir la côte qui mène au musée, etc. Il se fait le receleur d'informations stratégiques qu'il protège comme l'on gère un capital financier. Les analyses de Boissevain révèlent également que « le courtier peut toujours essayer, à un moment donné, de transformer ses ressources relationnelles de deuxième degré en ressources matérielles de premier degré: un poste dans l'administration, un terrain, de l'influence politique...» (Bierschenk T., Chauveau J.-P. \& Olivier de Sardan J.-P., 2000, p. 12). À terme, Dinoté Kusia brigue le poste de directeur du musée Konso : malgré les stratégies qu'il a mis en place pour l'obtenir, il sera finalement évincé pour des raisons politiques, mais surtout en raison des nombreuses histoires de personnes suscitées par sa nomination.

23 Le Kalla Gesahegn, quant à lui, dispose de pouvoirs religieux qui s'ajoutent à son pouvoir décisionnel qu'il n'hésite pas à mettre en avant : "My family is at the top of the clan, people do what I say, the others Poqalla follow me ». Il se trouve que le clan du Kalla Gesahegn occupe une place spéciale au sein de la société Konso et dispose de pouvoirs religieux qui se transmettent de père en fils aîné. En réalité, son importance est plus nuancée et varie dans le temps en fonction des événements historiques et de la capacité des Poqalla à accroître leur charisme (Demeulenaere, 2005, chapitre 9). Ainsi, cette position prestigieuse ne fait pas pour autant du Kalla Gesahegn le « roi » des Konso ${ }^{20}$ : les 9 Poqalla ont un statut égal et prennent leurs décisions de façon collégiale (Hallpike, 1972). Bien que le Kalla Gesahegn ne se présente pas comme un personnage politique, mais plutôt comme le détenteur d'un pouvoir spirituel, arguant qu'il y existe une nette différence entre son pouvoir naturel, "a power given by nature", et un pouvoir politique, il se plaint du manque de considération dont ferait preuve le Special wereda de Konso à son égard, relatant les conflits permanents qui les opposent concernant des affaires courantes aussi bien que des problèmes touchant à la représentation politique et à son pouvoir décisionnel. Selon lui, ils se méprennent sur son rôle au sein de la société Konso : le wereda l'exclut injustement et entretient une peur absurde quant à ses aspirations politiques ${ }^{21}$. Il explique que le wereda ne souhaitait pas sa présence à l'inauguration du musée, notamment en raison des conflits fonciers qui les opposent 
depuis des années ${ }^{22}$, et qu'il a fallu qu'une personnalité du Ministère des Affaires Fédérales éthiopien téléphone au Special wereda de Konso en intimant de l'écouter: «Listen to him ». Alors qu'il était étudiant à Addis-Abeba, il a pourtant été proche du parti. Lorsqu'il rentrait à Konso pour rendre visite à sa famille, il faisait des remarques au wereda sur les défaillances de sa politique en citant la nouvelle Constitution, notamment les droits culturels garantis aux minorités par le système ethno-fédéral. En 2005, il décide de quitter Addis-Abeba où il travaillait comme ingénieur civil pour devenir Poqalla suite au décès de son père. C'est son père justement qui l'aurait encouragé à faire des études, alors qu'il avait lui-même quitté les bancs de l'école avant même d'entrer au collège. Il est alors perçu comme un espion du gouvernement central, revenu de la ville afin de surveiller sa campagne natale. Selon lui, le fait qu'il revendique une légitimité à s'exprimer en raison d'une éducation nettement supérieure à la plupart des personnalités locales gêne le Special wereda, qui interpréterait cet état de fait comme une insulte aux fonctionnaires de l'administration éthiopienne. Cela lui aura surtout permis de travailler de façon rapprochée avec les équipes scientifiques du CFEE et du musée du Quai Branly et de s'affirmer comme un intermédiaire indispensable, puisqu'il est le seul chef de clan à maitriser parfaitement l'anglais. Dans ce contexte, le musée l'a aidé à se repositionner par rapport au milieu politique local en contribuant à le définir comme l'entité naturelle qu'il prétend être et non comme une personnalité politique. La duplicité dont il fait preuve se traduit par ses stratégies multiples pour que le lignage Kalla se trouve en position hégémonique par rapport aux autres : l'omniprésence de l'histoire de sa famille et de son clan dans les panels incite en effet le public à visiter son village ou à y passer la nuit, ce qui a amené Enrico Castelli, le directeur local de l'ONG CISS, à le présenter comme «l'invention même du tourisme " dans la région Konso. La muséographie lui a ainsi permis d'acquérir une légitimité exogène tout en évitant un conflit frontal avec le Special wereda. Au cours des années, il a donc développé un certain nombre de ressources lui permettant de s'instituer comme personne référente, bien que son objectif ne soit pas en premier lieu de se confronter aux autorités gouvernementales mais plutôt de s'octroyer un avantage symbolique en amenant les visiteurs à le considérer comme le porte-parole de la culture Konso (Geertz, 1983).

24 Si les conflits de pouvoirs locaux que véhiculent le musée prennent différentes formes - revendications violentes, provocations dans le cas de Dinoté Kusia ou processus d'infiltration de la muséographie et stratégies d'évitement du Kalla Gesahegn - ils nourrissent les débats de légitimité à l'échelle locale. Dans le même temps, ces acteurs sont parvenus à créer un discours identitaire structuré et figé faisant de la culture Konso une sorte de vérité anthropologique. Ainsi, le politique ou l'identité ne sont que des ressources parmi d'autres mobilisées par les acteurs de la vie locale pour peser sur le projet de musée. Raphaël Roig, qui a activement participé à l'établissement du musée à Konso, dresse une analyse plus large des motivations de ceux qu'on qualifie tantôt de courtiers, tantôt d' "entrepreneurs d'identité » (Martin, 2010) : "ces deux personnages sont au cœur du processus de cristallisation de la tradition et de la folklorisation d'une culture en voie d'extinction. Motivés par des intérêts personnels, ils défendent un retour aux mœurs traditionnelles Konso, une conservation et une mise en valeur d'un héritage culturel presque oublié. Dans un contexte local de transformations sociales profondes, ils prônent un retour en arrière vers une culture originelle, un nouveau paradis folklorique dont ils seront maîtres et gagnants. La création du musée Konso leur offrait un outil non négligeable pour arriver à leurs fins » (Roig, 2010, p. 295). Ainsi, il apparaît clairement que le musée Konso 
s'inscrit dans des enjeux de pouvoirs qui préexistaient à sa création : son apparition a créé un nouvel espace d'expression de critiques décomplexées, parfois virulentes, de la politique gouvernementale et des autorités locales.

\section{Le musée Konso, une « arène politique » locale à part entière}

25 En raison de la centralité des conflits au sein de ce projet, le musée Konso s'affirme comme une "arène politique " locale ${ }^{23}$ : comme le souligne les anthropologues du développement, " un projet de développement est une arène ", c'est-à-dire un «lieu de confrontations concrètes d'acteurs sociaux en interaction autours d'enjeux commun " (Bierschenk \& Olivier de Sardan, 1998, p. 262). Les conflits qui ont lieu dans cette arène mettent en relief des "groupes stratégiques" (mouvements religieux, agences de développement, chefs traditionnels, élus locaux) qui participent à la restructuration des relations de pouvoir en puisant des ressources à l'extérieur pour provoquer les reconfigurations locales dont ils ont besoin. Cette situation n'est pas nouvelle: auparavant, la coopération entre les autorités traditionnelles Konso et le wereda se faisait par le biais d'intermédiaires, comme l'ONG italienne CISS ou l'association PARKA pour la protection de l'environnement et de la culture Konso, qui participa à la rédaction du premier projet de classement UNESCO et dont le Kalla Gesahegn est membre. Ces structures tampons, qui font l'objet d'un contrôle strict par le régime éthiopien (cf. la loi sur les ONG de 2008), ne suffisent pas à absorber les chocs provoqués par les chevauchements de pouvoir entre les autorités administratives et claniques. Ainsi, l' " arène politique » locale constituée par le musée Konso a peut-être émergé en ce qu'elle pallie à ce manque : marquée par un trait principal qui est la polycéphalie du pouvoir, elle permet la confrontation directe de différentes autorités qui voient leur légitimité respective entrer en concurrence. C'est cette polycéphalie qui permet un contournement du centralisme et de l'autoritarisme d'État en favorisant l'« institution shopping» (Bierschenk \& Olivier de Sardan, 1998, p. 38), processus au cours duquel les différents acteurs de l'arène ont la possibilité de choisir parmi les organisations et les acteurs auxquels ils s'adressent en fonction de priorités et de stratégies qui leurs sont propres, ce que les entretiens avec Dinoté Kusia et le Kalla Gesahegn nous révèlent clairement. Ainsi, la politique s'effectue dans les «arènes politiques » locales sur un mode de "sédimentation » et de "chevauchement » entre des strates de pouvoir accumulées par une multitude d'acteurs dans le temps qui échappent au contrôle de l'État (Bierschenk \& Olivier de Sardan, 1998, p. 49). Ce que l'on pourrait nommer le $"$ champ politique $»^{24}$ éthiopien au sens de Pierre Bourdieu, c'est à dire l'espace autonome plus large créé par les compétitions que se livrent les agents sociaux afin d'y occuper des positions dominantes, se trouve modifié par l'apparition du musée Konso qui, en tant qu'arène, instaure de nouvelles personnalités « notables » cherchant à se (re)positionner autour du projet de musée.

26 Si l'«arène politique » locale, investie par les acteurs du développement et de la coopération internationale, a pour spécificité de provoquer un recul progressif du contrôle de l'appareil d'État (Bierschenk\& Olivier de Sardan, 1998), alors il est possible de percevoir les effets réels du glocal sur les articulations du pouvoir en Éthiopie. Ce constat rejoint des approches récentes en science politique qui utilisent le concept $d^{\prime}$ " « arène » comme une variable afin de décrire l'influence sur le processus décisionnel 
ou l'action publique des dispositifs mis en place par les courtiers pour se saisir d'une nouvelle "ressource" (Faure, 1997; Gourges, 2010). En ce sens, les intermédiaires nécessaires que sont les "courtiers en développement » pourraient avant tout être compris comme des «entrepreneurs politiques " puisqu'au-delà de leurs discours, ils sont les acteurs d'une « prise de pouvoir sur les affaires publiques du village » (LavigneDelville, 2008, p. 166).

27 En se faisant le catalyseur de nouveaux gains liés au développement (apparition de bénéfices financiers, d'emplois et de ressources diverses, etc.), le musée Konso constitue donc une arène à part entière. Loin du lieu commun qui décrit une situation dans laquelle l'accès au pouvoir et aux ressources économiques dépend étroitement de l'accès aux circuits de l'aide internationale et du contrôle de sa répartition, ce sont les stratégies de mobilisation ou de captation de cette rente provenant de l'aide au développement ${ }^{25}$ qui s'affirment comme des enjeux essentiels au niveau local, redessinant les contours du champ politique éthiopien dans son ensemble (Olivier De Sardan, 1993).

\section{Conclusion}

28 L'étude du musée Konso apporte une nouvelle lecture des enjeux de pouvoir en Éthiopie. Ses manquements tiennent à ce qu'il est investi d'intentions qui dépassent de loin les seuls problèmes structurels que peut poser la mise en place d'un projet culturel. Son statut $d$ '« arène politique » locale le met au centre de luttes interpersonnelles servant des intérêts individuels, mais aussi de luttes plus larges que sont la volonté de rayonnement d'institutions occidentales ou de contrôle de l'État central. L'offre patrimoniale, et plus particulièrement le musée Konso, fonctionne comme une « vitrine » colorée au service du gouvernement, exposant aux yeux de tous une Éthiopie forte de sa diversité, temporisant ainsi (ou tout du moins masquant partiellement) la «double crise » dont est victime l'État éthiopien depuis la mort du premier ministre Meles Zenawi en août 2012.

L'usage qui est ici fait de la culture s'apparente aux pratiques totalitaires analysées par Hannah Arendt, engageant un phénomène de disparition de l'individu dans une communauté essentialisante dont le musée pourrait être l'un des vecteurs. La synergie, non dénuée de contradictions, qui existe entre politiques gouvernementales, luttes locales et interventions extérieures souligne l'impact de l'idéologie développementaliste sur les représentations de la culture dans l'Éthiopie contemporaine. La nature de l'État éthiopien s'en trouve questionnée : État autoritaire, démocratie révolutionnaire, un "État néo-patrimonial» compris dans un sens nouveau, qui userait du patrimoine comme une nouvelle façon de construire sa légitimité, son rapport à la société et au monde extérieur ? (Médard, 1991) Si la fonction assignée aux musées dans la réinvention d'un imaginaire national (Anderson, 1991) et dans la construction d'une mémoire collective réutilisée à des fins politiques a été largement étudiée en Europe (Nora, 1986 ; Lavabre, 2000), ces questions ne peuvent trouver leurs réponses qu'au sein d'une analyse sur le temps long du rôle joué par ces derniers au sein de l'État développementaliste éthiopien.

30 Enfin, la forte intrication qui existe entre les entreprises de contrôle gouvernementales et les luttes locales pour le pouvoir et l'influence a permis l'émergence d'une catégorie d'acteur au rôle ambivalent: plus que d'être de simples 
intermédiaires entre des donateurs et des bénéficiaires potentiels de la coopération internationale, les "courtiers en développement" sont les porteurs sociaux et politiques des projets de développement locaux. En assurant l'interface entre les destinataires du projet et les institutions de développement, ils s'affirment comme des intermédiaires incontournables en développant des stratégies complexes afin de représenter la population locale ou d'exprimer ses besoins vis-à-vis des structures d'appui et de financement. Loin d'être les opérateurs passifs d'une logique d'assistanat, ils sont les personnages clés de l'articulation de pouvoir entre le local, le national et l'international. Sans poser sur des acteurs comme Dinoté Kusia ou le Kalla Gesahegn de présupposés normatifs, tant positifs - les courtiers comme émanation d'une société civile se dressant face à l'adversité - que négatifs - les courtiers comme profiteurs d'une aide mal maitrisée - la question de la «toute puissance» des courtiers en développement sur la mise en place de projets culturels, et donc, sur la représentation d'une identité, reste posée.

\section{BIBLIOGRAPHIE}

Aalen L., Pausewang S., Tronvoll K. (dir.), 2002. Ethiopia since the Derg, A Decade of Democratic Pretension and Performance. Zed Books, London, $245 \mathrm{p}$.

Aalen L., 2006. Ethnic Federalism and Self-determination for Nationalities in a Semi-authoritarian State: the case of Ethiopia. International Journal on Minority and Groups Rights, vol. 13, p. 246-261.

Abbink J., 2006. Discomfiture of democracy? The 2005 Election Crisis in Ethiopia and its Aftermath. African Affairs, vol. 105, n 419, p. 173-199.

Abbink J., Hagmann T. (dir.), 2013. Reconfiguring Ethiopia: the politics of authoritarian reform. Routledge, $232 \mathrm{p}$.

Anderson B., 1991. Imagined Communities: Reflections on the Origin and Spread of Nationalism. Verso, $224 \mathrm{p}$.

Asnake Kefale, 2009. Federalism and Ethnic Conflict in Ethiopia: a comparative study of the Somali and Benishangul-Gumuz Regions. Doctoral thesis, Leiden University.

Assefa Fiseha, 2006. Theory versus Practice in the Implementation of Ethiopia's Ethnic Federalism. In Turton David (dir.), Ethnic Federalism, Eastern African Studies, Addis Ababa University Press, p. 131-164.

Assefa Fiseha, 2012. Ethiopia's Experiment in Accommodating Diversity: 20 Years'Balance Sheet, Regional \& Federal Studies, 22:4, p. 435-473.

Bach J.-N., 2009. Chronique du Millénium éthiopien. Annales d'Éthiopie, Centre Français des Études Éthiopiennes, Addis-Abeba, Volume 24, p. 337-372.

Bach, J.-N., 2011. Centre, périphérie, conflit et formation de l'Etat depuis Ménélik II : Les crises "de" et "dans" l'Etat éthiopien (XIXe-XXIe siècles). Thèse de doctorat en Sciences politique, Les Afriques dans le Monde, Sciences Po Bordeaux. 
Bach J.-N., 2013 a. Abyotawi democracy: Neither revolutionary nor democratic: a critical review of EPRDF's conception of revolutionary democracy in post-1991 Ethiopia. In Abbink J. \& Hagmann T. (dir.), Reconfiguring Ethiopia: the politics of authoritarian reform, Routledge, 2013, p. 62-84.

Bach J.-N., 2013 b. Compromising with Ethiopianness after 1991: The Ethiopian Festival of the Millennium (September 2007-September 2008). Northeast African Studies, Special Issue, Guidi P. \& Vezzadini H.(dir.), vol. 13, n², p. 93-122.

Bierschenk T. et Olivier De Sardan J.-P. (dir.), 1998. Les pouvoirs aux villages, le Bénin rural entre démocratisation et décentralisation. Karthala, 296 p.

Bierschenk T., Chauveau J.-P., Olivier de Sardan J.-P., 2000. Courtiers en développement. Les villages africains en quête de projet. Mayence, APAD, Institut für Ethnologie, J. Gutemberg Universität, Paris, Karthala, 328 p.

Boissevain J., 1974. Friends of Friends. Networks, Manipulators and Coalitions. Oxford, Blackwell.

Bosc-Tiessé C., 2009. How to write Art History in Konso: Stakes and Orientations of Research. communication au séminaire du CFEE, 15 décembre 2009.

Bourdieu P., 1993. Esprits d'État. Actes de la recherche en sciences sociales, vol. 96-97, p. 49-62.

Bridonneau M., 2013. Lalibela, une petite ville d'Éthiopie amarrée au monde : analyse des recompositions spatiales, sociales et politiques dans une petite ville patrimoniale, sacrée et touristique. Thèse de Doctorat en géographie, Université Paris 10.

Bureau J., 1985. L'espace politique éthiopien. Annales ESC, novembre-décembre 1985, p. 1379-1393.

Camau M., Geisser V., 2003. Le syndrome autoritaire : politique en Tunisie de Bourguiba à Ben Ali. Presses de Sciences Po, $372 \mathrm{p}$.

Chaudron M., 2008. Le patrimoine mondial de l'humanité, un état des lieux 1972-2006. In J.-P. Vallat (dir.), Mémoires de patrimoines. L'Harmattan, p. 171-186.

Debub Negarit Gazeta, 2011. The Southern Nations, Nationalities and People's Regional State Konso Cultural Landscape Heritages Conservation Proclamation. Journal officiel de la SNNPR Proclamation $\mathrm{n}^{\circ} 141 / 2011$.

Demeulenaere E., 2005. Herbes folles et arbres rois, gestion paysanne des ligneux au pays Konso (Éthiopie), contribution à la définition d'un patrimoine naturel. Thèse en Ethnobiologie au MNHN.

Faure A., 1997. Les apprentissages du métier d'élu local : la tribu, le système et les arènes. Pôle Sud, $\mathrm{n}^{\circ}$ 7, p. 72-79.

Gascon A., 1995. La grande Éthiopie, une utopie africaine. CNRS éditions, coll. Espaces \& Milieux, $256 \mathrm{p}$.

Gascon A., 2008. Shining Ethiopia : l'Éthiopie post-communiste du nouveau millénaire. Autrepart, 4/ 2008, nº 48, p. 141-152.

Gaugue A., 1997. Les États africains et leurs musées. La mise en scène de la nation. Paris, L'Harmattan, coll. Géographie et Cultures, $232 \mathrm{p}$.

Geertz C., 1983. Centres, rois et charisme : réflexions sur les symboliques du pouvoir. In Savoir local, savoir global : les lieux du savoir. Paris, PUF, p. 153-182.

Gourgues G., 2010. Le consensus participatif. Les politiques de la démocratie dans quatre régions françaises. Thèse de science politique, Université de Grenoble. 
Hallpike C.-R., 1972. The Konso of Ethiopia. The studies of the Values of Cushitic Peoples. Clarendon Press, Oxford University Press, Oxford, 342 p.

Lamarque P., Demeulenaere E., 2010. Le roi ne meurt jamais. Lussas, DocNet Films, DVD 73 min.

Lavabre M.-C., 2000. Usages et mésusages de la notion de mémoire. Critique internationale, Vol. 7, p. $48-57$.

Lavigne-Delville P., 2000. Courtiers en développement ou entrepreneurs politiques? Les responsables d'associations villageoises de développement dans une région d'émigration internationale. In Bierschenk T., Chauveau J.-P., Olivier de Sardan J.-P., (dir.), Courtiers en développement. Les villages africains en quête de projet. Mayence, APAD, Institut für Ethnologie, J. Gutemberg Universität, Paris, Karthala, p. 165-188.

Lefort R., 2013. The Theory and Practice of Meles Zenawi: A Response to Alex de Waal. African Affairs, 112 (448), p. 460-70.

Le Hugon P., 2009. L'économie de l'Afrique. La découverte, 128 p.

Martin D.-C., 2010. L'identité en jeux. Pouvoirs, identifications, mobilisations. Karthala, coll. Recherches internationales, $466 \mathrm{p}$.

Médard J.-F., 1990. Autoritarismes et démocratie en Afrique. Politique africaine, $\mathrm{n}^{\circ} 43$.

Médard J.-F., 1991. L'État néo-patrimonial en Afrique noire, États d'Afrique Noire : formations, mécanismes et crises. Paris, Karthala, 323 p.

Mengistu Gobezie, 2008, Heritage tourism in Ethiopia, imprimé à compte d'auteur, Addis-Abeba, $300 \mathrm{p}$.

Merera Gudine, 2006. Contradictory Interpretations of Ethiopian History: The Need for a New Consensus. In Turton D. (dir.), Ethnic Federalism, Eastern African Studies, Addis-Ababa University Press, Addis-Ababa, p. 199-129.

Nallet C., en cours. Classe.s. Moyenne.s. Éthiopienne.s. : une catégorie de référence fantasmée, des réalités sociales plurielles structurantes. Thèse de doctorat en Science politique, Les Afriques dans le Monde, Institut d'Études Politiques de Bordeaux.

Nallet C., 2014. Quelle sucess story pour l'Éthiopie ? Décryptages, IFRI, Paris, https:// afriquedecryptages.wordpress.com/2014/06/05/quelle-success-story-pour-lethiopie/

Nora P., 1986. Les lieux de Mémoire. Gallimard, Paris, 3 tomes (t.1 La République, t.2 La Nation, t. 3 Les France).

Olivier De Sardan J-P., 1993. Le développement local comme champ politique local. Bulletin de l'APAD, 6, Marseille, p. 11-18.

Otto Shako, 2005. Traditionnal Konso Culture and the Missionary Impact. Les Annales d'Éthiopie, vol. 20, p. 149-180.

Planel S., 2007. Du centralisme à l'ethno-fédéralisme. La décentralisation conservatrice de l'Ethiopie. In Le Bris E., Paulais T., Décentralisations et développements, Afrique Contemporaine, $\mathrm{n}^{\circ} 221$, p. 87-105.

Planel S., 2008. La chute d'un Eden éthiopien : le Wolaita, une campagne en recomposition. IRD, Marseille, $430 \mathrm{p}$.

Planel S., De Poix S., 2009. Les justices de l'État en Éthiopie. Lieux, époques et institutions du juste et de l'injuste. Annales de Géographie, n 665-666, Armand Colin, p. 138-156. 
Roig R., 2010. Chronique du musée Konso. Une brève analyse des enjeux de la coopération et de la politique culturelle en Région Konso. Annales d'Éthiopie, n² 25, p. 283-300.

Vaughan S., 2006. Responses to Ethnic Federalism in Ethiopia's Southern Region. In Turton D. (dir.), Ethnic Federalism, Eastern African Studies, Addis-Ababa University Press, Addis Ababa, James Currey, Oxford, p. 181-207.

Vestal T. M., 1999. Ethiopia : A post-cold war African State. Praeger, 229 p.

\section{NOTES}

1. Au-delà du musée Konso lui-même, cet engouement s'est traduit en Éthiopie par plusieurs projets similaires : l'ouverture en 2010 du Red Terror Martyr's Memorial Museum dédié à la période du Derg, la restructuration des bâtiments du Musée National d'Addis-Abeba et la création d'un parcours historique dans ses jardins au cours de l'année 2012, ainsi que l'ouverture prochaine d'un musée historique à Gondar. À ce sujet, voir également l'étude menée par Anne Gaugue sur les musées africains et la mise en scène de la nation (Gaugue, 1997).

2. Le wereda est une subdivision administrative regroupant un ensemble de qébélé (unité de voisinage proche du quartier). Si l'on tentait d'établir une comparaison avec le système français, le wereda serait plus ou moins équivalent à une communauté de communes ou à un district selon le système anglo-saxon.

3. J.-F Médard et J.-F Bayart utilisent le terme de « décompression autoritaire » afin de relativiser la libéralisation des régimes autoritaires africains supposés entamer leur transition démocratique dans les années 1990, qui s'adaptent alors aux revendications de la société civile sans mettre en place une réelle libéralisation du système politique. Ces derniers font effet preuve d'une capacité d'adaptation largement décrite par les analyses de Camau et Geisser sur la Tunisie (Camau \& Geisser, 2003).

4. Le terme "Renaissance» est employé lors des discours officiels pour désigner à la fois une nouvelle ère politique, économique et sociale à laquelle s'ouvre l'Éthiopie contemporaine.

5. Il faut noter que le Millénium éthiopien s'est déroulé au cours de l'année 2007 du calendrier européen. En effet, 7 ou 8 ans séparent le calendrier européen du calendrier éthiopien, construit sur le modèle copte qui varie selon le nombre de jours épagomènes par année.

6. René Lefort considère la classe moyenne éthiopienne comme la nouvelle "darling child of the regime ", tandis que Clélie Nallet montre dans sa thèse que les groupes sociaux intermédiaires constituent un nouvel enjeu privilégié par le gouvernement (Lefort, 2013; Nallet, thèse de doctorat en Science politique, en cours).

7. Le successeur de Meles Zenawi à la position de Premier Ministre et souvent considéré comme le « dauphin » de ce dernier, Hailé Mariam Dessalegn, originaire du Sud, est un bon exemple de cette politique.

8. Marie Bridonneau analyse par exemple comment la promotion de l'écotourisme, notion importée mais centrale dans le discours de certains acteurs locaux, participe de l'internationalisation de la ville de Lalibela en Éthiopie (Bridonneau, 2013, p. 269).

9. Un tukul est le terme amharique qui désigne une hutte ronde construite en bois et en terre et couverte d'un toit de paille.

10. Selon la comptabilité établie par le Bureau de la Culture du Special wereda consultée par l'auteur en novembre 2012, le musée Konso a accueilli 2192 visiteurs de décembre 2011 à novembre 2012, parmi lesquels 1372 touristes étrangers et 820 touristes éthiopiens. Ces visites représentent un chiffre d'affaire de 28068 birr (monnaie éthiopienne) pour 2012, soit environ 1220 euros. Le tarif de base est de 20 birr pour les visiteurs étrangers et éthiopiens, et de 50 birr par personne lorsqu'il s'agit d'une visite de groupe. 
11. Suite à la création du musée à Konso-karat, une association de guides locaux, pour la plupart de jeunes hommes originaires de la région, a été formée. Atta (qui signifie «culture» en konsigna), dispose d'un petit bureau d'où partent les visites et fonctionne en corrélation étroite avec le Bureau de la culture, qui délivre les tickets autorisant les visites des villages fortifiés Konso classés au patrimoine mondial de l'UNESCO.

12. L'argument d'une « mise en scène » se justifie par un certain nombre de dysfonctionnements du musée Konso évoqués ci-dessus, qui révèlent avant tout que ce musée n'a pas pour vocation d'être une institution locale comme il avait pourtant prétention à l'être. Cette idée se retrouve dans la proclamation $n^{\circ} 141 / 2011$ du journal officiel de la SNNPR, le Debub Negarit Gazeta, dont l'article 15.3 stipule que plus largement, le patrimoine culturel Konso «shall be promoted, visited and used for building the image of the country ".

13. En 2003, l'Éthiopie hébergeait 419 ONG, alors qu'elle en accueillait seulement 47 en 1989. Face à ce développement effréné qui ne permettait plus au gouvernement de les contrôler sur le sol éthiopien, une loi restreignant leur capacité d'action a été votée en 2008. Elles sont dorénavant soumises à des réglementations comptables et administratives qui peuvent condamner leurs dirigeants à de lourdes peines (jusqu'à 15 ans de prison) en cas de non-respect des procédures. Les ONG dites «étrangères " (financement étranger supérieur à $10 \%$ ) voient leur champ d'intervention réduit puisqu'il leur est désormais interdit d'agir dans les secteurs tels que le droit des femmes, des enfants et des handicapés, le fonctionnement de la justice ou la résolution des conflits.

14. Le terme gäbar (qui signifie «tributaire » en amharique) désigne les tenanciers précaires ou " paysans sans terre » du Sud de l'Éthiopie qui étaient dans l'obligation de verser des redevances matérielles ou financières à un seigneur (goult) selon un rapport de force proche de celui mis en place par le système féodal européen.

15. Un Special wereda est un échelon administratif accordé à un groupe ethnique sur les bases de son importance démographique dans une région donnée. En théorie, le Special wereda se voit attribuer la même autonomie administrative qu'une zone au sein du système fédéral.

16. Rappelons ici que Poqalla est un terme konsigna désignant un chef de clan Konso, quand le terme de Kalla désigne un linage de Poqalla. Ces statuts sont décrits avec précision par le documentaire réalisé par Pierre Lamarque et Élise Demeulenaere, "Le roi ne meurt jamais ", 2010, Lussas, DocNet Films, DVD 73 min.

17. Les propos rapportés ici sont issus de plusieurs entretiens semi-directifs menés par l'auteur à différents moments de mon enquête. Elle a pu interroger par deux fois le Kalla Gesahegn : à Konso-Karat tout d'abord, à la terrasse du café principal du village en présence de Dinoté Kusia et du responsable de la culture du Special wereda. C'est lors de ce premier entretien qu'il cherche à naturaliser sa fonction pour assoir sa légitimité et ne pas attiser les conflits avec le Special wereda. Le deuxième entretien se déroule à Addis-Abeba alors qu'il était venu participer à une conférence sur le thème de la santé : accompagné de son jeune frère, il parle alors plus en détail de son pouvoir décisionnel et se confie sur les relations difficiles qu'il entretient avec le Special wereda. Plusieurs entretiens avec Dinoté Kusia ont eu lieu au cours de la recherche sur le site du musée Konso, chez lui et au café principal de Konso-Karat. Ces entretiens se déroulent toujours sur le mode de la « confidence ", ce qui correspond à une stratégie de Dinoté Kusia afin de mettre en scène son importance et sa dissidence vis-à-vis du Special wereda et de solliciter l'aide de l'auteur après chaque discussion.

18. Dinoté Kusia fut il y a dix années de cela l'un des premiers guides associés au Bureau de la Culture de Konso-Karat.

19. Il est important de préciser que l'auteur a toujours appartenu à la catégorie des chercheurs, tout d'abord en tant que membre de l'équipe du CFEE en charge du projet de musée puisque qu'elle y effectuait un stage de 11 mois (septembre 2008-juillet 2009), en tant qu'étudiante de master ensuite (février 2010) puis en tant que doctorante (novembre 2012). Elle était donc à la 
fois proche de ses interlocuteurs en raison de ses visites régulières à Konso-Karat, et en même temps considérée comme un acteur du projet à part entière, ce qui a pu parfois orienter les discours que ces derniers tenaient en sa présence lors des situations observées comme lors des entretiens individuels.

20. S'il n'utilise jamais le terme de « roi » lors de cérémonies officielles, il laisse les touristes l'utiliser à son égard. Stéphane Gompertz, alors Ambassadeur de France en Éthiopie, le présente en tant que tel lors de l'inauguration du musée Konso en décembre 2009, provoquant de vives réactions dans l'assemblée (Roig, 2010, p. 295).

21. Ces tensions datent en fait de l'époque où son père Woldedawit officiait en tant que Kalla: on raconte qu'il avait obtenu un titre de noblesse de la part de l'Empereur Hailé Sellassié, qui serait venu lui rendre visite par hélicoptère dans la forêt. C'est précisément ces stratégies multiples, sur deux générations, qui font que le lignage Kalla se retrouve actuellement en position hégémonique par rapport aux autres lignages de Poqalla. Par ailleurs, le Kalla Gesahegn affirme lors d'un entretien qu'en réalité, les membres officiels du wereda ont peur de représailles de la part des autorités claniques en raison des mauvais traitements que le gouvernement central leur aurait fait subir par le passé.

22. Le Kalla est un propriétaire terrien par définition, il est tout particulièrement responsable de la forêt sacrée, de la gestion des ressources de la communauté, alors que le wereda, selon la loi, est le détenteur légal de ces terres (Demeulenaere, 2005). À ce sujet, voir les analyses de Bierschenk et Olivier de Sardan sur l'influence des chefs «traditionnels » sur le foncier (Bierschenk \& Olivier De Sardan, 1998, p. 34).

23. Le concept $d$ '« arène politique » locale s'inscrit dans le champ de l'anthropologie dynamique développée par G. Balandier et des approches d'A. Giddens du changement social dites « orientées vers les acteurs ». Ce concept sera privilégié à la notion de « champ politique " parce qu'il est plus approprié au contexte du musée Konso au sein duquel les différents acteurs en interaction permanente soulignent précisément une absence de cloisonnement entre politique et culture contraire à l'autonomie des champs culturel et politique telle qu'elle est définie par Bourdieu.

24. Pierre Bourdieu comprend le monde social comme divisé en ce qu'il nomme des « champs ». Il lui semble, en effet, que la différenciation des activités sociales a conduit à la constitution de sous-espaces sociaux, comme le champ artistique ou encore le champ politique, spécialisés dans l'accomplissement d'une activité sociale donnée. Ces champs, dotés d'une autonomie relative les uns par rapport aux autres, se forment grâce aux luttes, aux compétitions que se livrent les agents sociaux afin d'y occuper des positions dominantes (Bourdieu, 1993).

25. Par «aide au développement» on entend toutes les formes d'aides qui aujourd'hui constituent une réalité massive pour beaucoup de pays africains: aide au développement "publique et, de plus en plus, décentralisée, non gouvernementale ou privée ; don, prêt bonifié ou emprunt sur le marché privé international ; aide bilatérale ou multilatérale » (Le Hugon, 2009).

\section{RÉSUMÉS}

À partir de l'exemple du musée Konso, premier musée ethnographique au Sud de l'Éthiopie, cet article décrit les usages, détournements et récupérations par le gouvernement éthiopien et par diverses figures locales - ou «courtiers en développement »- d'un projet né de la coopération 
culturelle. La reconfiguration des échelles et des rapports de pouvoir en périphérie de l'Éthiopie fédérale amène une réflexion nécessaire sur l'aspect patrimonial des politiques publiques éthiopiennes ainsi que sur les acteurs de la légitimation de l'idéologie développementaliste du parti au pouvoir.

This paper aims to enlighten the current powergames and scales at stake within the Federal Democratic Republic of Ethiopia from a local perspective. The role of heritage policies and specific actors of the development field in legitimating the ideology of the Developmental State is questionned in relation to the Konso Museum, the first Ethiopian single-Museum dedicated to an ethnic group of the Southern Ethiopia. This case-study underlines the multiple use of an international cooperation project in showcasing the liberalization of the Regime.

\section{INDEX}

Keywords : heritage, international cooperation, Developmental State, Konso museum, ethnicfederalism, Southern Nations, Nationalities and Peoples' Region

Mots-clés : patrimoine, coopération internationale, État développementaliste, musée Konso, fédéralisme ethnique, Southern Nations, Nationalities and Peoples' Region

\section{AUTEUR}

\section{CHLOÉ JOSSE-DURAND}

Chloé Josse-Durand, chloe.ethiopie@gmail.com, Doctorante en Science Politique au laboratoire Les Afriques dans le Monde, Institut d'Etudes Politiques de Bordeaux. 